\title{
Stability Analysis of the Transmission Dynamic and Control of Infectiousrate under Treatment
}

\author{
D. Jasmine \\ PG \& Research Department of Mathematics \\ Bishop Heber College, Tiruchirappalli, Tamil Nadu, India
}

\begin{abstract}
On account of the effect of limited treatment resources on the control of epidemic disease, the transmission dynamic and control of infectious rate under treatment is incorporated. The stability analysis of the disease-free equilibrium and the endemic equilibrium are discussed with treatment rate.
\end{abstract}

Keywords: Basic Reproduction Number, Disease-free equilibrium, Endemic equilibrium

\section{Introduction}

Kermack and McKendrick divided the total population $\left(P_{t}\right)$ into three compartments such as susceptible $\left(S_{t}\right)$, Infective $\left(I_{t}\right)$ and Removable $\left(R_{t}\right)$ individuals, so that:

$P_{t}=S_{t}+I_{t}+R_{t}(1)$

Where

$\frac{d S_{t}}{d t}=i S_{t} I_{t}(2)$

$\frac{d I_{t}}{d t}=i S_{t} I_{t}-g I_{t}(3)$

$\frac{d R_{t}}{d t}=g I_{t}(4)$

\section{Model Formulation}

In this model, we divide the total population into four compartments such as Susceptible, Infective, Hospitalized and Recovered individuals. We assume that:

- $S$ is the number of susceptible individuals who are not infected but could become infected.

- Iis the number of infective individuals who are infected by the disease and can transmit it to the susceptible.

- $\mathrm{H}$ is the number of individuals who are admitted in the hospital to take treatment for a specified period.

- $R$ is the number of recovered or removed individuals These may or may not have the disease, but they can't become infected and they can't transmit the disease to others.

A susceptible, infective or hospitalized individual can willingly become recovered or removed.

The model variables and parameters are defined as follows

$N(t)$ Total population individual at time $\mathrm{t}$
$S(t)$ Susceptible individual at time $\mathrm{t}$

$I(t)$ Infective individual at time $\mathrm{t}$

$H(t)$ Hospitalized individual at time $\mathrm{t}$

$\mathrm{R}(t)$ Recovered individual at time $\mathrm{t}$

$A$ Recruitment rate of the population

$\mu$ Death removal rate

$p$ Infection transmission probability per contact

$\tau$ Effort rate against infection and thus

$\beta=p(1-\tau)$ is the effective infectious contact

rate

$\phi$ Rate at which infective individual are admitted as a patient

$\frac{1}{\psi}$ Average period of the infected individual spent in the hospital

$\theta$ Proportion of individuals that leaves $\mathrm{S}$, I or $\mathrm{A}$ compartment to $\mathrm{R}$

The susceptible sub-population $\mathrm{S}(\mathrm{t})$ is generated from constant recruitment of individual at a rate $A$. They acquired infection via horizontal transfers from individual in the infective class, $\mathrm{I}(\mathrm{t})$ at a rate $\beta=p(1-\tau)$ and thus become infective individual in the susceptible infective and admitted class become removed due to treatment on the danger of infection at the rate $\theta(0<\theta<1)$. Infective individuals are admitted and treated at the rate $\emptyset$ admitted individuals stay in hospital for an average period of $\frac{1}{\psi}$ after which a proportion $\theta$ become recovery while (1- $\theta)$ go back to infective class and not due to the fear of the consequence of being admitted. Thus as the admitted individual spent an average period of $\frac{1}{\psi}$ in the hospital then $\Psi \theta$ and $\Psi(1-\theta)$ are the transition rates from $\mathrm{H}$ compartment to $\mathrm{R}$ compartment and back to $\mathrm{I}$ compartment respectively.

Furthermore, natural death occurs in all classes at a rate ?. The corresponding mathematical equation of model can be

Volume 5 Issue 6, June 2016 www.ijsr.net 


\section{International Journal of Science and Research (IJSR) \\ ISSN (Online): 2319-7064}

Index Copernicus Value (2013): 6.14 | Impact Factor (2015): 6.391

described by a system of ordinary differential equations given in (5)

$$
\begin{aligned}
& \frac{d S}{d t}=A-\frac{\beta I}{N} S-K_{1} S \\
& \frac{d I}{d t}=\frac{\beta I}{N} S+\psi(1-\theta) H-K_{2} I \\
& \frac{d H}{d t}=\phi I-K_{3} H+T(I) \\
& \frac{d R}{d t}=\theta(S+I+\psi H)-\mu R-T(I)
\end{aligned}
$$

where $T(I)=r I$. Now

$\frac{d S}{d t}=A-\frac{\beta I}{N} S-K_{1} S$

$\frac{d I}{d t}=\frac{\beta I}{N} S+\psi(1-\theta) H-K_{2} I$

$\frac{d H}{d t}=\phi I-K_{3} H+r I$

$\frac{d R}{d t}=\theta(S+I+\psi H)-\mu R-r I$

$K_{1}=(\theta+\mu)$

$K_{2}=(\phi+\theta+\mu)(6)$

$K_{3}=(\psi+\mu)$

Consider the closed set:

$\Omega=\left\{(S, \mathrm{I}, H, \mathrm{R}) \in: S+I+H+R \leq \frac{A}{\mu}\right\}(7)$

In order to study the dynamics of the system (5) in $\Omega$, the positive-invariance and attractiveness of $\Omega$ with respect to the system(5) is established as follows now the rate of change of the total population, obtained by adding all the equation in the system (5) is given by

$\frac{d N}{d t}=A-\mu N$ (8) It follows from (8) that whenever $N>\frac{A}{\mu}$ then $\frac{d N}{d t}=0$ implying $\frac{d N}{d t}$ is bounded by $A-\mu N$. Thus a standard comparison theorem can be used to show that $N(t) \leq N(0) e^{-\mu t}+\frac{A}{\mu}\left(1-e^{-\mu t}\right)$, thus $\Omega$ is positively-invariant (i.e. all solution in $\Omega$ remain in $\Omega$ for all time).

Furthermore, if $N(t)>\frac{A}{\mu}$ then either the solution enters $\Omega$ in finite time or $\mathrm{N}(\mathrm{t})$ approaches $\frac{A}{\mu}$ and the infected variables (i.e., all solution in eventually enters $\Omega$ ).
Therefore the model is well-posed epidemiologically and mathematically (Hetthcote, 2000). And hence it is sufficient to study the dynamics of the system (5) in $\Omega$.

\section{Model Analysis}

\subsection{Existence and Local Stability of Disease-Free Equilibrium}

The disease-free equilibrium is the state in which the population is free of disease, so that we have only susceptible and recovered individuals. Thus the model has a disease-free equilibrium, obtained by setting the righthand side of (5) to zero given by

$A-\frac{\beta I}{N} S-K_{1} S=0 \&$ Put I=0, we get

$S=\frac{A}{K_{1}} \quad \& \quad R=\frac{\theta(S+I+\psi H)-r I}{\mu}$

Put $\mathrm{H}=0 \Rightarrow R=\frac{\theta S}{\mu}$

i.e., $R=\frac{\theta A}{\mu K_{1}}$

$E_{0}:\left(S^{*}, \mathrm{I}^{*}, H^{*}, \mathrm{R}^{*}\right)=\left(\frac{A}{K_{1}}, 0,0, \frac{\theta A}{\mu K_{1}}\right)(9)$

Using the next generation operator technique described by Diekmann and Heesterbeek (2000) and subsequently analyzed by Van Den Driessche and Watmough (2002), we obtained the basic reproduction number $R_{0}$ of the model equation (5) which is the spectral radius $(\rho)$ of the next generation matrix $\mathrm{K}$.

That is $R_{0}=\rho K$, where $K=F V^{-1}$ the matrix of F (for the new infection terms) are obtained from the infected compartment (i.e., I and $\mathrm{H}$ ) at disease-free equilibrium and are given respectively by

$$
\begin{aligned}
& \frac{d S}{d t}=A-\frac{\beta I}{N} S-K_{1} S \\
& \frac{d H}{d t}=\phi I-K_{3} H+r I \\
& F=\left(\begin{array}{cc}
-\frac{\beta S}{N} & 0 \\
\phi+r & -K_{3}
\end{array}\right) \\
& F=\frac{\beta K_{3} S^{*}}{N^{*}}
\end{aligned}
$$




$$
\begin{aligned}
& \frac{d I}{d t}=\frac{\beta I}{N} S+\psi(1-\theta) H-K_{2} I \\
& \frac{d H}{d t}=\phi I-K_{3} H+r I \\
& V=\left(\begin{array}{cc}
-K_{2} & \psi(1-\theta) \\
\phi+r & -K_{3}
\end{array}\right) \\
& V=K_{2} K_{3}-(\phi+r) \psi(1-\theta) \\
& F=\left(\begin{array}{cc}
\frac{\beta S^{*}}{N^{*}} & 0 \\
-(\phi+r) & K_{3}
\end{array}\right)(10)
\end{aligned}
$$

and

$V=\left(\begin{array}{ll}K_{2} & -\psi(1-\theta) \\ -(\phi+r) & K_{3}\end{array}\right)$

The basic reproduction number is then given as:

$$
\begin{aligned}
& R_{0}=F K=F V^{-1}=\frac{F}{V} \\
& R_{0}=\frac{\beta K_{3} S^{*}}{\left(K_{2} K_{3}-(\phi+r) \psi(1-\phi)\right) N^{*}}
\end{aligned}
$$

\section{Theorem:}

The disease-free equilibrium $E_{0}$ of the model is locally asymptotically stable (LAS) if $R_{0}<1$.

\section{Proof.}

We used the Jacobean stability approach to prove the local stability of the disease-free equilibrium state. Now, we observed that the variable $R$ does not appear in the first three (3) equations of the system (5).

Thus, using the relation

$$
\begin{aligned}
& S+I+H+R=N \\
& R=N-(S+I+H)
\end{aligned}
$$

Linearization at $E_{0}$, gives the Jacobian matrix:

$$
\begin{aligned}
& \frac{d S}{d t}=A-\frac{\beta I}{N} S-K_{1} S \\
& \frac{d I}{d t}=\frac{\beta I}{N} S+\psi(1-\theta) H-K_{2} I \\
& \frac{d H}{d t}=\phi I-K_{3} H+r I
\end{aligned}
$$

$$
H\left(E_{0}\right)=\left(\begin{array}{ccc}
-K_{1} & -\frac{\beta S^{*}}{N^{*}} & 0 \\
0 & -\left(K_{2}-\frac{\beta S^{*}}{N^{*}}\right) & \psi(1-\theta) \\
0 & \phi+r & -K_{3}
\end{array}\right)
$$

Considering (5) at $E_{0}$, we can deduced that

$$
\begin{aligned}
& \frac{d S}{d t}=A-\frac{\beta I}{N} S-K_{1} S \\
& \frac{d I}{d t}=\frac{\beta I}{N} S+\psi(1-\theta) H-K_{2} I \\
& \frac{d H}{d t}=(\phi+r) I-K_{3} H \\
& \frac{d R}{d t}=\theta(S+I+\psi H)-\psi R-r I
\end{aligned}
$$

$$
\begin{aligned}
& A-\frac{\beta I^{*}}{N}-K_{1} S^{*}=0 \\
& \Rightarrow A=\frac{\beta I^{*} S^{*}}{N^{*}}+K_{1} S^{*} \\
& \frac{\beta I^{*}}{N^{*}} S^{*}+\psi(1-\theta) H^{*}-K_{2} I^{*}=0 \\
& \Rightarrow K_{2}=\frac{\beta S^{*}}{N^{*}}+\frac{\psi(1-\theta) H^{*}}{I^{*}} \\
& (\phi+r) I^{*}-K_{3} H^{*}=0 \\
& \Rightarrow K_{3}=\frac{(\phi+r) I^{*}}{H^{*}} \\
& \theta\left(S^{*}+I^{*}+\mu H^{*}\right)-\mu R^{*}-r I^{*}=0 \\
& \Rightarrow \mu=\frac{\theta\left(S^{*}+I^{*}+\mu H^{*}\right)-r I^{*}}{R^{*}}
\end{aligned}
$$

Therefore

$$
\begin{aligned}
& A=\frac{\beta I^{*} S^{*}}{N^{*}}+K_{1} S^{*} \\
& K_{2}=\frac{\beta S^{*}}{N^{*}}+\frac{\psi(1-\theta) H^{*}}{I^{*}} \\
& K_{3}=\frac{(\phi+r) I^{*}}{H^{*}} \\
& \mu=\frac{\theta\left(S^{*}+I^{*}+\psi H^{*}\right)-r I^{*}}{R^{*}}
\end{aligned}
$$

Using elementary row transformation, equation (14) becomes

\section{Volume 5 Issue 6, June 2016}




$$
\begin{aligned}
& R_{1} \rightarrow R_{1} \\
& R_{2} \rightarrow R_{2} \\
& R_{3} \rightarrow R_{3}\left(K_{2}-\frac{\beta S^{*}}{N^{*}}\right)+(\phi+r) R_{2} \\
& H\left(E_{0}\right)=\left[\begin{array}{ccc}
-K_{1} & -\frac{\beta S^{*}}{N^{*}} & 0 \\
0 & -\left(\mathrm{K}_{2}-\frac{\beta S^{*}}{N^{*}}\right) & \psi(1-\theta) \\
0 & \phi & -K_{3}+\frac{(\phi+r) \Psi(1-\theta)}{\left(\mathrm{K}_{2}-\frac{\beta S^{*}}{N^{*}}\right)}
\end{array}\right]
\end{aligned}
$$

And clearly, the eigen values are

$$
\begin{aligned}
& \lambda_{1}=-K_{1}<0 \\
& \lambda_{2}=-\left(K_{2}-\frac{\beta S^{*}}{N^{*}}\right)<0
\end{aligned}
$$

Since from equation (15)

$$
\begin{aligned}
& K_{2}=\frac{\beta S^{*}}{N^{*}}+\frac{\psi(1-\theta) H^{*}}{I^{*}} \\
& K_{2}>\frac{\beta S^{*}}{N^{*}} \\
& \text { and } K_{2} N^{*}-\beta S^{*}>0 \\
& \lambda_{3}=-\left(K_{3}-\frac{(\phi+r) \psi(1-\theta) N^{*}}{K_{2} N^{*}-\beta S^{*}}\right)
\end{aligned}
$$

Now $\lambda_{3}$ to be negative, we must have

$$
\begin{aligned}
& -K_{3}\left(K_{2} N^{*}-\beta S^{*}\right)+(\phi+r) \psi(1-\theta) N^{*}<0 \\
& \text { i.e., } \beta K_{3} S^{*}<K_{3} K_{2} N^{*}-(\phi+r) \psi(1-\theta) N^{*} \\
& \frac{\beta K_{3} S^{*}}{\left(K_{2} K_{3}-(\phi+r) \psi(1-\theta) N^{*}\right.}<1
\end{aligned}
$$

Thus $\lambda_{3}<0$ if $R_{0}<1$ implying all the eigen values have negative real parts and by Jacobean stability $\mathrm{E}_{0}$ is LAS. This completes the proof.

The epidemiological implication of the theorem is that infection can be under control in the population (when $\mathrm{R}_{0}<$ 1) if the initial sizes of the sub-populations of the model are in the basis of attraction of DFE $\left(\mathrm{E}_{0}\right)$. In order to ensure that corruption is independent of the initial sizes of the sub-populations of the model, it is necessary to show that $\mathrm{E}_{0}$ is globally-asymptotically stable.

\subsection{Global Stability of Disease-Free Equilibrium}

\section{Theorem:}

The disease-free equilibrium $E_{0}$ of (5) is globally asymptotically stable (GAS) in $\Omega$ if $R_{0} \leq 1$.

\section{Proof.}

One common approach in studying the global asymptotic stability of the DFE is to construct an appropriate Lyapunov function. Consider the Lyapunov function

$$
L=K_{3} I+\psi(1-\theta) H(18)
$$

It's derivative along the solutions of the model equation is

$$
\begin{aligned}
& L^{\prime}=K_{3} I^{\prime}+\psi(1-\theta) H^{\prime} \\
& =K_{3}\left(\frac{\beta I}{N} S+\psi(1-\theta) H-K_{2} I\right) \\
& \quad+\psi(1-\theta)\left[(\phi+r) I-K_{3} H\right] \\
& =K_{3} I\left(\frac{\beta S}{N}\right)-I\left[K_{2} K_{3}-(\phi+r) \psi(1-\theta)\right] \\
& =I\left[K_{2} K_{3}-(\phi+r) \psi(1-\theta)\right] \\
& \quad \mathrm{X}\left(\frac{K_{3} \beta S}{\left[K_{2} K_{3}-(\phi+r) \psi(1-\theta)\right] N}-1\right)
\end{aligned}
$$

Since $\frac{S}{N} \leq \frac{S^{*}}{N^{*}}$, we have

$$
\begin{aligned}
L^{\prime} \leq I\left[K_{2} K_{3}-(\phi+r) \psi(1-\theta)\right] \\
\mathrm{X}\left(\frac{K_{3} \beta S^{*}}{\left[K_{2} K_{3}-(\phi+r) \psi(1-\theta)\right] N^{*}}-1\right)
\end{aligned}
$$

i.e., $L^{\prime} \leq I\left[K_{2} K_{3}-(\phi+r) \psi(1-\theta)\right]\left(R_{0}-1\right)$ where

$$
R_{0}=\frac{K_{3} \beta S^{*}}{\left[K_{2} K_{3}-(\phi+r) \psi(1-\theta)\right] N^{*}} \text { (19) }
$$

Since all model parameters are non-negative and from (15), we have

$$
\begin{aligned}
& K_{2}=\frac{\beta S^{*}}{N^{*}}+\frac{\psi(1-\theta) H^{*}}{I^{*}} \\
& K_{3}=\frac{(\phi+r) I^{*}}{H^{*}} \\
& K_{2} K_{3}=\left(\frac{\beta S^{*}}{N^{*}}+\frac{\psi(1-\theta) H^{*}}{I^{*}}\right)\left(\frac{(\phi+r) I^{*}}{H^{*}}\right) \\
& =\frac{\beta S^{*}(\phi+r) I^{*}}{N^{*} H^{*}}+\frac{(\phi+r) \psi(1-\theta) H^{*} I^{*}}{H^{*} I^{*}} \\
& K_{2} K_{3}=\frac{\beta S^{*} I^{*}(\phi+r)}{N^{*} H^{*}}+(\phi+r) \psi(1-\theta) \\
& K_{2} K_{3}>(\phi+r) \psi(1-\theta)
\end{aligned}
$$

It follows that when $R_{0} \leq 1, L^{\prime} \leq 0$, the equality is zero holds when $R_{0}=1$ and $\mathrm{I}=0$.

Therefore, the largest compact invariant set $\left\{(S, \mathrm{I}, H, \mathrm{R}) \in L^{\prime}=0\right\}$ is the singleton $\left\{\mathrm{E}_{0}\right\}$. Hence, by the LaSalle invariance principle, $\mathrm{E}_{0}$ is overall globally asymptotically stable and hencethe proof is complete. 


\section{International Journal of Science and Research (IJSR) \\ ISSN (Online): 2319-7064}

Index Copernicus Value (2013): 6.14 | Impact Factor (2015): 6.391

The above theorem shows that infection will be under control regardless of the initial profile of the subpopulation in the community if $\mathrm{R}_{0}$ can be brought down to a level less than unity.

\subsection{Existence of Disease-Endemic Equilibrium}

At the disease endemic equilibrium we have persistence of infection. Thus at least one of the infected class is greater than zero. In order to find the positive endemic equilibrium of the system (5), denoted by

$$
E^{*}=\left(S^{* * *}, \mathrm{I}^{* *}, H^{* *}, \mathrm{R}^{* *}\right)
$$

The equations in the system (5) are solved as explained in Gumel (2007) in terms of the associated force of infection at steady-state, given by

$$
\begin{aligned}
& \lambda^{* *}=\frac{\beta I^{* * *}}{N^{* *}}(21) \\
& A-\frac{\beta I^{* *}}{N^{* *}} S^{* *}-K_{1} S^{* *}=0 \\
& \Rightarrow S^{* * *}=\frac{A}{K_{1}+\lambda^{* * *}} \\
& \lambda^{* * *} S^{* * *}+\psi(1-\theta) H^{* *}-K_{2} I^{* *}=0 \\
& \frac{\lambda^{* *} A}{K_{1}+\lambda^{* *}}+\frac{(\phi+r) \psi(1-\theta) I^{* *}}{K_{3}}-K_{2} I^{* *}=0 \\
& \frac{\lambda^{* *} A}{K_{1}+\lambda^{* *}}=\left(K_{2}-\frac{(\phi+r) \psi(1-\theta)}{K_{3}}\right) I^{* *} \\
& \frac{\lambda^{* *} A}{K_{1}+\lambda^{* * *}}=\left(\frac{K_{2} K_{3}-(\phi+r) \psi(1-\theta)}{K_{3}}\right) I^{* *}
\end{aligned}
$$

$$
I^{* *}=\frac{\lambda^{* *} A K_{3}}{\left(K_{1}+\lambda^{* *}\right)\left(K_{2} K_{3}-(\phi+r) \psi(1-\theta)\right.}
$$$$
(\phi+r) I^{* *}=K_{3} H^{* *}
$$$$
H^{* *}=\frac{A(\phi+r) \lambda^{* *}}{\left(K_{1}+\lambda^{* * *}\right)\left[K_{2} K_{3}-(\phi+r) \psi(1-\theta)\right]}
$$$$
\mu R^{* * *}=\theta\left(S^{* * *}+I^{* *}+\psi H^{* * *}\right)-r I^{* *}
$$$$
\begin{aligned}
\mu R^{* *} & =\theta\left\{\frac{A}{K_{1}+\lambda^{* * *}}+\frac{\lambda^{* *} A K_{3}}{\left(K_{1}+\lambda^{* *}\right)\left[K_{2} K_{3}-(\phi+r) \psi(1-\theta)\right]}\right. \\
& \left.+\frac{\psi A(\phi+r) \lambda^{* *}}{\left(K_{1}+\lambda^{* *}\right)\left[K_{2} K_{3}-(\phi+r) \psi(1-\theta)\right]}\right\}-\frac{\lambda^{* *} A K_{3} r}{\left(K_{1}+\lambda^{* *}\right)\left[K_{2} K_{3}-(\phi+r) \psi(1-\theta)\right]}
\end{aligned}
$$$$
R^{* *}=\frac{A \theta\left[K_{2} K_{3}-(\phi+r) \psi(1-\theta)\right]+\left\{A \theta\left[K_{3}-(\phi+r) \psi\right]-A r K_{3}\right\} \lambda^{* *}}{\mu\left(K_{1}+\lambda^{* *}\right)\left[K_{2} K_{3}-(\phi+r) \psi(1-\theta)\right]}
$$

Solving the equations of the model (5) at steady-state gives

$$
\begin{aligned}
& S^{* *}=\frac{A}{K_{1}+\lambda^{* *}} \\
& I^{* *}=\frac{\lambda^{* *} A K_{3}}{\left(K_{1}+\lambda^{* *}\right)\left[K_{2} K_{3}-(\phi+r) \psi(1-\theta)\right]} \\
& H^{* *}=\frac{\lambda^{* *} A(\phi+r)}{\left(K_{1}+\lambda^{* *}\right)\left[K_{2} K_{3}-(\phi+r) \psi(1-\theta)\right]} \\
& R^{* *}=\frac{A \theta\left[K_{2} K_{3}-(\phi+r) \psi(1-\theta)\right]+\left\{A \theta\left[K_{3}-(\phi+r) \psi\right]-A r K_{3}\right\} \lambda^{* *}}{\mu\left(K_{1}+\lambda^{* *}\right)\left[K_{2} K_{3}-(\phi+r) \psi(1-\theta)\right]}
\end{aligned}
$$

Using the second equation of system (22) in (21) and simplifying it gives

$$
\begin{aligned}
& I^{* * *}=\frac{\beta K_{3} A}{N^{* *}\left(K_{1}+\lambda^{* *}\right)\left[K_{2} K_{3}-(\phi+r) \psi(1-\theta)\right]} \\
& K_{1}+\lambda^{* * *}=\frac{\beta K_{3} A}{N^{* *}\left[K_{2} K_{3}-(\phi+r) \psi(1-\theta)\right]} \\
& \lambda^{* *}=\frac{\beta K_{3} A}{N^{* * *}\left[K_{2} K_{3}-(\phi+r) \psi(1-\theta)\right]}-K_{1} \\
& \lambda^{* *}=K_{1}\left(\frac{\beta K_{3}}{\mathrm{~K}_{1}\left[K_{2} K_{3}-(\phi+r) \psi(1-\theta)\right] \mathrm{N}^{* *}}-1\right) \\
& \text { i.e., } \lambda^{* *}=K_{1}\left(R_{0}-1\right)(23)
\end{aligned}
$$

Since all model parameters are assumed and non-negative with $\mu>0$, it follows that $\lambda^{* *}>0$, whenever $\mathrm{R}_{0}>1$. The components of $\mathrm{E}^{*}$ are then determined by substituting (23) into (22), given by: 


\section{International Journal of Science and Research (IJSR) \\ ISSN (Online): 2319-7064}

Index Copernicus Value (2013): 6.14 | Impact Factor (2015): 6.391

$$
\begin{aligned}
S^{* * *}= & \frac{A}{K_{1}+K_{1}\left(R_{0}-1\right)}=\frac{A}{K_{1} R_{0}}=\frac{A}{K_{1} R_{0}} \\
I^{* * *}= & \frac{\left(R_{0}-1\right) A K_{3}}{R_{0}\left[K_{2} K_{3}-(\phi+r) \psi(1-\theta)\right]} \\
H^{* * *}= & \frac{A(\phi+r)\left(R_{0}-1\right)}{R_{0}\left[K_{2} K_{3}-(\phi+r) \psi(1-\theta)\right]} \\
& \begin{aligned}
&\left(A \theta \left\{\left[K_{2} K_{3}-(\phi+r) \psi(1-\theta)\right]\right.\right. \\
& R^{* * *}= \frac{\left.\left.+\left[K_{3}-(\phi+r) \psi\right]\right\}-A r K_{3}\right)\left(R_{0}-1\right)}{\mu R_{0}\left[K_{2} K_{3}-(\phi+r) \psi(1-\theta)\right]}
\end{aligned}
\end{aligned}
$$

Noting that $\mathrm{R}_{0}<1$ implies that the force of infection at steady state $\left(\lambda^{* *}\right)$ negative (which is biologically meaningless). Hence the model has no positive equilibria in this case. Thus, we established the following result.

\subsection{Local Stability of Disease Endemic Equilibrium}

Similarly, as in local stability of disease-free equilibrium, we used the Jacobian stability approach to prove the stability of the disease endemic equilibrium state. Noting the relation $\mathrm{R}=N-(S+I+H)$, the Jacobian matrix of system (5) at $\mathrm{E}^{*}$ is given by

$$
H\left(E^{*}\right)=\left(\begin{array}{ccc}
-\left(\frac{\beta I^{* *}}{N^{* * *}}+K_{1}\right) & -\frac{\beta S^{* *}}{N^{* *}} & 0 \\
\frac{\beta I^{* *}}{N^{* * *}} & -\left(K_{2}-\frac{\beta S^{* *}}{N^{* *}}\right) & \psi(1-\theta) \\
0 & \phi+r & -K_{3}
\end{array}\right)
$$

From (5) at $\mathrm{E}^{*}$, we have

$$
\begin{aligned}
& A=\frac{\beta I^{* *} S^{* *}}{N^{* *}}+K_{1} S^{* *} \\
& K_{2}=\frac{\beta S^{* *}}{N^{* *}}+\frac{\psi(1-\theta) H^{* *}}{I^{* *}} \\
& K_{3}=\frac{(\phi+r) I^{* *}}{H^{* *}} \\
& \mu=\frac{\theta\left(S^{* *}+I^{* *}+\psi H^{* *}\right)-r I^{* *}}{R^{* *}}
\end{aligned}
$$

Using elementary row operation (25) becomes:

$$
H\left(E^{*}\right)=\left(\begin{array}{ccc}
-\left(\frac{\beta I^{* *}+K_{1} N^{* *}}{N^{* *}}\right) & -\frac{\beta S^{* *}}{N^{* *}} & 0 \\
0 & -M & \psi(1-\theta) \\
0 & 0 & -K_{3}+\frac{(\phi+r) \psi(1-\theta)}{M}
\end{array}\right)
$$

where $M=\left(K_{2}-\frac{\beta S^{* *}}{N^{* *}}+\frac{\beta^{2} S^{* *} I^{* *}}{\left(\beta I^{* *} K_{1} N^{* *}\right) N^{* * *}}\right)$
(28)

Thus, clearly the eigen values are:

$$
\lambda_{1}=-\left(\frac{\beta I^{* *}+K_{1} N^{* *}}{N^{* *}}\right)<0
$$

Since from (26) $K_{2}>\frac{\beta S^{* *}}{N^{* *}}$ and

$$
\lambda_{3}=-\left(K_{3}-\frac{(\phi+r) \psi(1-\theta)}{M}\right)(29)
$$

Now for $\lambda_{3}$ to be negative, we must have

$$
\frac{-K_{3} M+(\phi+r) \psi(1-\theta)}{M}<0
$$

i.e., $K_{3}\left\{K_{2}-\frac{\beta S^{* *}}{N^{* *}}+\frac{\beta^{2} S^{* *} I^{* *}}{\left(\beta I^{* *}+K_{1} N^{* *}\right.}\right\}>(\phi+r) \psi(1-\theta)$

Simplifying this, we obtain $\beta I^{* *}>0$

Substituting (24) and simplifying, we obtain $\left(R_{0}-1\right)>0$.

Thus $\lambda_{3}<0$. If $\mathrm{R}_{0}>1$ implies that, all the eigen values has negative real parts.

\section{Conclusion}

A mathematical model with constant recruitment rate and standard incidence for the transmission dynamics of infection as a disease was proposed. The basic reproduction number $\left(\mathrm{R}_{0}\right)$ was obtained and the analysis revealed that for $\mathrm{R}_{0} \leq 1$, the disease-free equilibrium is globally asymptotically stable. Although, the illicit and secretive nature of infection can never allow for its total eradication, but it can be curbed (reduced) to a bearable level. And for whatever reason if $\mathrm{R}_{0}>1$ the disease-free equilibrium point is unstable and the endemic equilibrium emerges.

\section{References}

[1] Capasso V and SerioG : A Generalization of the Kermack-Mckindrick Deterministic Epidemic Model, Math, Bio.Sci.42 (1978), 43-61.

[2] Diekmann O. and Heesterbeek J. A. P. (2000). Mathematical Epidemiology of Infectious Diseases, Model building analysis and interpretation, New York, John Wiley \& Sons, London.

[3] GajendraUjjainkar, Gupta V.K, Singh B, KhandeludR : An Epidemic Model with Modified Non-monotonic Incidence Rate under Treatment, Vol. 6 (2012) 11591171.

[4] Gumel A. B. (2004). Modelling strategies for controlling SARS outbreaks, Proc. R. Soc. London, B 271, 2223-2232.

[5] Hethcote H. W. (2000). The mathematics of infectious diseases. SIAM review, 42, 599-653.

[6] Kar T.K and AshimBatabyal : Modeling and Analysis of an Epidemic Model with Non-monotonic Incidence Rate under Treatment, Journal of Mathematical Research Vol.2, No. 1, (2010) 103-115. 


\section{International Journal of Science and Research (IJSR) \\ ISSN (Online): 2319-7064}

Index Copernicus Value (2013): 6.14 | Impact Factor (2015): 6.391

[7] Ruan S and Wang W: Dynamical Behavior of an Epidemic Model with Non-linear Incidence Rate. J.Differential Equations, 188, (2003) 135-163.

[8] Van den P. Driessche and Watmough J. (2002). Reproduction numbers and sub-threshold endemic equilibria for compartmental models of disease transmission, Math. Biosc., 180, 29-48.

[9] Wang W: Backward Bifurcation of an Epidemic Model with Treatment, J. Math, Bio. Sci. 201, (2006) $58-71$.

[10] Xiao D and Ruan S : Global Analysis of an Epidemic Model with Non-monotone Incidence Rate. Math.Bio.Sci. 208, (2007) 419-429

Volume 5 Issue 6, June 2016 www.ijsr.net 